TRANSACTIONS OF THE

AMERICAN MATHEMATICAL SOCIETY

Volume 355, Number 10, Pages 4045-4059

S 0002-9947(03)03183-0

Article electronically published on June 18, 2003

\title{
GENERALIZED HYPERELLIPTIC SURFACES
}

\author{
FRANCESCO ZUCCONI
}

\begin{abstract}
This article presents some results on the surfaces of general type whose Albanese morphism is a holomorphic fibre bundle.
\end{abstract}

\section{INTRODUCTION}

A fibration $f: S \rightarrow B$ is a surjective morphism between complete smooth varieties $S, B$ with connected fibres. The quotient surface by a diagonal action of a group $G$ over the product of two curves gives a typical example of important fibrations for the theory of surfaces. Perhaps the hyperelliptic surfaces are among the most interesting cases; their first Betti number $b_{1}$ is 2 and they have a holomorphically locally trivial fibration over an elliptic curve ([B] $]$, or cf. [BPV], pp. 148 and 189). In other words, a hyperelliptic surface $S$ is a quotient $S=E \times C / G$ where $E$ and $C$ are elliptic curves and $G$ is a finite subgroup of the translation group of $C$ which acts faithfully on $E$ with quotient isomorphic to $\mathbb{P}^{1}$.

In this paper we describe quotient surfaces $S=C_{1} \times C_{2} / G$ by the diagonal action of a finite group $G$ over $C_{1} \times C_{2}$ such that $i$ ) the Galois morphism $\pi_{1}: C_{1} \rightarrow C=C_{1} / G$ is unramified; $\left.i i\right)$ the quotient curve $C_{2} / G$ is isomorphic to $\mathbb{P}^{1}$. They are called Generalized-Hyperelliptic surfaces, GH in short. By the standard inequalities among the invariants of a fibration it follows that GH-surfaces are precisely those surfaces whose Albanese morphism is a holomorphic fibre bundle. Recently Catanese $\mathrm{Ca}$ ] has strongly renewed this subject showing their topological characterization: [Ca Theorem B]. The main theorem of this paper concerns another aspect of GH-theory.

Consider a fibration $f: S \rightarrow \mathbb{P}^{1}$ and let $\mathcal{E} \subset f_{\star} \mathcal{O}_{S}\left(K_{S}\right)$ be the rank $r_{f}$ subsheaf generically generated by the global sections of $f_{\star} \mathcal{O}_{S}\left(K_{S}\right)$. We call $r_{f}$ the rank of the fibration $f$. In fact, $r_{f}-1$ is the dimension of the linear series obtained by the restriction of the canonical linear series to a fibre.

Theorem A. Let $f: S \rightarrow \mathbb{P}^{1}$ be a rank- $r_{f}>0$ fibration on an arbitrary surface of general type. Assume that the space of global holomorphic 1-forms has dimension $r_{f}+1$. Then the Albanese image of $S$ is a curve if and only if the minimal model of $S$ is a GH-surface.

We also study the relation of $G H$-geometry with finite group theory. It is not difficult to prove that if $G$ is a finite group, then there exists a GH-surface $S$ such

Received by the editors February 2, 2001 and, in revised form, June 12, 2002.

2000 Mathematics Subject Classification. Primary 14J29, 14J25, 14 D06.

Key words and phrases. Fibration, Galois theory, canonical map.

This work was done at DPMMS-Cambridge, and it was partly supported by UK node of EAGER (European algebraic geometry research training network), EU contract RTN1-1999-00202. 
that $S=C_{1} \times C_{2} / G$ (see 4.3) ), and for each occurrence of $G$ we have plenty of GH-surfaces. However, the important point is to understand how $G$ influences the geometry of $S$. This can be seen in the next two classification theorems, especially in the last one.

The first theorem ends the classification of surfaces not of Albanese general type with canonical map composed with a pencil and with $q \geq 2$.

Theorem B. Let $S$ be a surface of general type with $q \geq 2$ such that its canonical map is composed with a pencil $\Lambda$ of curves of genus $h$. If its Albanese image is a curve, then $S$ is of Generalized Hyperelliptic type. Moreover, there are only two cases: a) $h=9, p_{g}=q=2$, or $\left.b\right) p_{g} \geq 2, S$ is a $G$-quotient of $X=C_{1} \times C_{2}$ where $G=\mathbb{Z} / 2, C_{1}$ is also the fiber of the canonical morphism and it has genus $h=3, C_{2}$ is the fiber of the Albanese morphism, it is a hyperelliptic curve of genus $p_{g}$, and $G$ acts on $\mathrm{C}_{2}$ via the hyperelliptic involution.

This theorem is essentially due to Konno. In fact, in [ $\mathrm{K}$ Theorem 3.6] it is proved that there is only the case $b)$ and possibly the cases $\left(p_{g}, h\right)=(2,6),(2,9),(3,7)$. Our proof is simple, and we can exclude the cases $\left(p_{g}, h\right)=(2,6),(3,7)$. Actually case $b$ ) has also been described in [Be2, Example 2].

In the second theorem we explicitly classify all GH-surfaces whose canonical image is a ruled surface with ruling induced by the natural pencil $f: S \rightarrow \mathbb{P}^{1}$ over a GH-surface. We think that this theorem is interesting because the group $G$ plays the main role. Moreover, in its proof we will need to consider also the nonabelian covers.

Let $G^{\star}$ be the set of the linear characters of $G$. Consider the corresponding decompositions $H^{0}\left(\Omega_{C_{1}}^{1}\right)=\bigoplus_{\chi \in G^{\star}} V_{(i)}^{\chi}, H^{0}\left(\Omega_{C_{2}}^{1}\right)=\bigoplus_{\chi \in G^{\star}} W_{(i)}^{\chi}$ into subspaces induced by the admissible faithful representations $G \rightarrow \operatorname{Aut}\left(C_{1}\right), G \rightarrow \operatorname{Aut}\left(C_{2}\right)$ where $i=\operatorname{dim} V_{(i)}^{\chi}=\operatorname{dim} W_{(i)}^{\chi}$ and where $G$ acts on $V_{(i)}^{\chi}$ and $W_{(i)}^{\chi}$ via $\chi$.

Theorem C. If $S=C_{1} \times C_{2} / G$ is a $G H$-surface such that the canonical image is birational to $\mathbb{P}^{1} \times\left(C_{2} / G\right)$ and the $\mathbb{P}^{1}$ 's are embedded as lines, then $G$ is abelian and only a finite list of cases occurs. More precisely, $S$ is constructed by the data given in Table 1.

TABLE 1.

\begin{tabular}{|c|c|c|c|c|}
\hline$q(S)$ & $G$ & $g_{2}=g\left(C_{2}\right)$ & $H^{0}\left(\Omega_{C_{2}}^{1}\right)$ & $H^{0}\left(\Omega_{S}^{2}\right)$ \\
\hline 3 & $\mathbb{Z} / 2$ & $\geq 2$ & $W_{(g)}^{-}$ & $V_{(2)}^{-} \otimes W_{(g)}^{-}$ \\
\hline 2 & $\mathbb{Z} / 3$ & $\geq 3$ & $W_{(i)}^{\chi} \oplus W_{(l-i)}^{\chi^{2}}$ & $V_{1}^{\chi} \otimes W_{(l-i)}^{\chi^{2}} \oplus V_{1}^{\chi^{2}} \otimes W_{(i)}^{\chi}$ \\
\hline 2 & $\mathbb{Z} / 4$ & 3 & $W_{1}^{\chi^{2}} \oplus W_{(2)}^{\chi^{3}}$ & $V_{1}^{\chi} \otimes W_{(2)}^{\chi^{3}} \oplus V_{1}^{\chi^{(2)}} \otimes W_{1}^{\chi^{2}}$ \\
\hline 2 & $\mathbb{Z} / 4$ & $2 l-3 \geq 3$ & $W_{(l-1)}^{\chi} \oplus W_{(l-2)}^{\chi^{3}}$ & $V_{1}^{\chi} \otimes W_{(l-2)}^{\chi^{3}} \oplus V_{1}^{\chi^{3}} \otimes W_{(l-1)}^{\chi}$ \\
\hline 2 & $\mathbb{Z} / 4$ & $2 l-2 \geq 4$ & $W_{(l-1)}^{\chi} \oplus W_{(l-1)}^{\chi^{3}}$ & $V_{1}^{\chi} \otimes W_{(l-1)}^{\chi^{3}} \oplus V_{1}^{\chi^{3}} \otimes W_{(l-1)}^{\chi}$ \\
\hline 2 & $\mathbb{Z} / 2 \times \mathbb{Z} / 2$ & $2 l-1 \geq 3$ & $W_{(l-1)}^{\chi_{2}} \oplus W_{(l-1)}^{\chi_{1} \chi_{2}}$ & $V_{1}^{\chi_{2}} \otimes W_{(l-1)}^{\chi_{2}} \oplus V_{1}^{\chi_{1} \chi_{2}} \otimes W_{(l-1)}^{\chi_{1} \chi_{2}}$ \\
\hline 2 & $\mathbb{Z} / 2 \times \mathbb{Z} / 2$ & $2 l-2 \geq 4$ & $W_{(l-1)}^{\chi_{2}} \oplus W_{(l)}^{\chi_{1} \chi_{2}}$ & $V_{1}^{\chi_{2}} \otimes W_{(l-1)}^{\chi_{2}} \oplus V_{1}^{\chi_{1} \chi_{2}} \otimes W_{(l)}^{\chi_{1} \chi_{2}}$ \\
\hline
\end{tabular}


Finally we would like to remind the reader the paper of Serrano [Ser] where we learned about the concept of isotrivial fibred surfaces and where the reader can find the concept of quasi-bundle, which includes the surfaces we are going to study.

\section{First RESUlts ON GH-SURFACES}

To write our results, we have to recall some basic facts about fibration theory of surfaces. The first one is that if the fiber $F$ has genus $g>0$, then $f$ admits a unique minimal model (called minimal fibration) and it is obtained by successive contraction of the -1-curves contained in the fibers. To study $f: S \rightarrow B$, we denote by $b$ and $g$ the genus of $B$ and $F$ respectively. The standard notation for the dimension of the space of holomorphic 1-forms, and 2-forms is, respectively, $q=\operatorname{dim} H^{0}\left(S, \Omega_{S}^{1}\right)$ and $p_{g}=\operatorname{dim} H^{0}\left(S, K_{S}\right)$. We shall have to consider the following relative invariants:

$$
\begin{aligned}
& e_{f}=e(S) \quad-4(g-1)(b-1), \\
& \chi_{f}=\chi(S)-(b-1)(g-1), \\
& k_{f}=K_{S}^{2}-8(g-1)(b-1),
\end{aligned}
$$

where $e(S)$ is the topological Euler-Poincaré characteristic, $\chi(S)=p_{g}-q+1$ is the algebraic characteristic, and $K_{S}^{2}$ is the self-intersection number of the canonical class $K_{S}$ of $S$. Much information on $f$ is given by the invariants of the relative canonical sheaf $\omega_{S \mid B}=\mathcal{O}_{S}\left(K_{S}\right) \otimes f^{\star} \mathcal{T}_{B}$ where $\mathcal{T}_{B}$ is the tangent sheaf on $B$. In particular, it is known that $f_{\star} \omega_{S \mid B}$ is a rank- $g$ vector bundle and

$$
\operatorname{deg} f_{\star} \omega_{S \mid B}=\chi_{f} .
$$

The following known theorems are the cornerstones of the theory.

Theorem of Zeuthen-Segre. If $f: S \rightarrow B$ is a fibration, then $e_{f} \geq 0$. If $g \geq 2$, then $e_{f}=0$ if and only if $f$ is a topological fibre bundle.

Theorem of Fujita. If $f: S \rightarrow B$ is a minimal fibration with $g \geq 2$, then $f_{\star} \omega_{S \mid B}=\mathcal{O}_{B}^{\oplus q-b} \oplus \mathcal{F}$ where $\mathcal{F}$ is a nef locally free sheaf such that $h^{0}\left(B, \mathcal{F}^{\vee}\right)=0$. In particular, $\chi_{f} \geq 0$. Moreover, the equality $\chi_{f}=0$ holds if and only if $f$ is an analytic bundle.

Theorem of Arakelov. If $f: S \rightarrow B$ is a minimal fibration with $g \geq 2$, then $k_{f} \geq 0$ and $\omega_{S \mid B} C \geq 0$ for every irreducible curve $C \subset S$. Moreover, $k_{f}=0$ only if the smooth fibres of $f$ are isomorphic.

Obviously, if $f$ is a holomorphic fibre bundle, then all the fibres are smooth and isomorphic. In particular, the relative Todd genus formula $12 \chi_{f}=k_{f}+e_{f}$ and the equality $\chi_{f}=0$ in Fujita's theorem force the other two equalities $k_{f}=0, e_{f}=0$ to hold, and the natural questions are the following: I) For which surfaces is the canonical map a holomorphic fibre bundle? II) For which surfaces is the Albanese map a holomorphic fibre bundle? In particular, in this second case the surface is not of Albanese general type; that is, the image of the Albanese morphism is a curve. By $[\mathrm{X}]$, the answer to the first question is extremely easy in the case of surfaces of general type with $q \geq 2$ : none. To answer the second question we came across the concept of Generalized Hyperelliptic surface (see also [Ca, Proposition $3.15])$.

Let $C_{1}, C_{2}$ be two smooth curves, and $\operatorname{Aut}\left(C_{1}\right)$, Aut $\left(C_{2}\right)$ the associated automorphisms groups. We consider a nontrivial finite subgroup $G$ with two injections: $G \hookrightarrow \operatorname{Aut}\left(C_{1}\right), G \hookrightarrow \operatorname{Aut}\left(C_{2}\right)$. 
Definition 2.1. The quotient surface $S=C_{1} \times C_{2} / G$ by the diagonal action of $G$ onto the product $C_{1} \times C_{2}$ is said to be of Generalized Hyperelliptic type (GH, for short) if $i$ ) the Galois morphism $\pi_{1}: C_{1} \rightarrow C=C_{1} / G$ is unramified, and $i i$ ) the quotient curve $C_{2} / G$ is isomorphic to $\mathbb{P}^{1}$.

From now on $S=C_{1} \times C_{2} / G$ will always be a surface of Generalized Hyperelliptic type, unless otherwise stated. Note that $S$ is always a minimal surface. $S$ comes equipped with two different, important maps. The fibration with general fiber $C_{2}$ will be denoted by $\alpha: S \rightarrow C$ while the one with fiber $C_{1}$ will be denoted by $f: S \rightarrow \mathbb{P}^{1}$. The numbers $g_{1}, g_{2}$ will denote the genera of $C_{1}, C_{2}$.

Lemma 2.2. The Albanese map factors through the fibration $\alpha: S \rightarrow C$.

Proof. Trivial by the universal property of the Albanese morphism and Be1, Lemme VI.11].

We point out that $C$, the image of the Albanese morphism, is the quotient of $C_{1}$ by $G$. The first of our results actually is Theorem $\mathrm{F}$ in $\mathrm{Ca}$, where it is shown that among the fibred surfaces not of Albanese general type with $K_{S}^{2}=8 \chi(S)$, the GH are those with maximal genus of the fiber for the Albanese map. We present here an independent proof.

Proposition 2.3. Let $S$ be a surface with $q \geq 2$. The following are equivalent:

1) $S$ is a GH-surface;

2) the Albanese map induces a fibration $\alpha: S \rightarrow C$ with $\chi_{\alpha}=0$.

Proof. Suppose that the Albanese map is factored by a fibration $\alpha: S \rightarrow C$. By Fujita's theorem applied to $\alpha$, we know that $\chi_{\alpha} \geq 0$, and equality holds if and only if $\alpha$ is a holomorphic fibre bundle. Now if $\alpha$ is a holomorphic fibre bundle, by [Ser] we have $S=C_{1} \times C_{2} / G$, and $G \subset \operatorname{Aut}\left(C_{1}\right) \cap \operatorname{Aut}\left(C_{2}\right)$ acts freely on $C_{1}$. We have two cases. If $G$ acts diagonally, then $C_{2} / G=\mathbb{P}^{1}$; otherwise, by the universal property of the Albanese map it would be true that $C=C_{1} / G$ is a covering of $C_{2} / G$, a contradiction. Let us consider the case $C_{1}=C_{2}=A$ such that $G / G_{\text {fix }}=\mathbb{Z} / 2$, where $G_{\text {fix }}$ is the subgroup whose elements do not interchange the two factors in $X=A \times A$, and we have

$$
\begin{aligned}
H^{0}\left(\Omega_{S}^{1}\right) & =\left(H^{0}\left(\Omega_{A}^{1}\right) \oplus H^{0}\left(\Omega_{A}^{1}\right)\right)^{G} \subset H^{0}\left(\Omega_{A}^{1}\right)^{G_{\mathrm{fix}}} \oplus H^{0}\left(\Omega_{A}^{1}\right)^{G_{\mathrm{fix}}} \\
& =H^{0}\left(\Omega_{D}^{1}\right) \oplus H^{0}\left(\Omega_{D}^{1}\right) .
\end{aligned}
$$

The group $G / G_{\text {fix }}=\mathbb{Z} / 2$ interchanges the two factors $H^{0}\left(\Omega_{D}^{1}\right)$, and by the very nature of the Albanese morphism the following diagram is commutative:

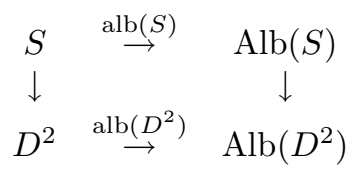

But $\operatorname{alb}\left(D^{2}\right)$ is the Abel-Jacobi map on the degree-2 divisor over $D$ and so its image is 2-dimensional. In particular, the image of $\operatorname{alb}(S)$ is a two-dimensional variety, a contradiction. Vice versa, if $S$ is GH, then $\alpha$ is a fibre bundle, and by Fujita's theorem we easily conclude. 
As a corollary we obtain an answer to question $I I)$ :

Theorem 2.4. The Albanese morphism of a surface of general type $S$ with $q \geq 2$ is a holomorphic bundle if and only if $S$ is of Generalized Hyperelliptic type.

Proof. Trivial by Fujita's theorem and Proposition 2.3.

Proposition 2.3 implies that in the GH-geometry the converse of Arakelov's theorem holds. In fact, it is an easy consequence of the Zeuthen-Segre, Fujita and Arakelov theorems stated in the introduction:

Proposition 2.5. Let $S$ be a surface with a constant moduli fibration $f: S \rightarrow B$ over a base of genus $b>0$. If $S$ is a GH-surface, then $k_{f}=0$.

Proof. Since $S$ is GH and $b>0$, then $f=\alpha$. In particular, $\chi_{f}=0$ by 2.3 On the other hand, by the Zeuthen-Segre and Arakelov theorems, we have $e_{f} \geq 0$ and $k_{f} \geq 0$. Now the claim follows from the relative version of Noether's theorem: $12 \chi_{f}=k_{f}+e_{f}$.

\section{THEOREM A}

We have studied the Albanese map of GH-surfaces. Let us consider the other fibration naturally living over $S, f: S \rightarrow \mathbb{P}^{1}$. We can associate to $f$ the subsheaf $\mathcal{E} \hookrightarrow f_{\star} \omega_{S}$ generically generated by the global sections. By Fujita's theorem and the well-known Grothendieck decomposability result for vector bundles on $\mathbb{P}^{1}$ we have

$$
f_{\star} \omega_{S}=\mathcal{O}_{\mathbb{P}^{1}}^{\oplus q(S)}(-2) \oplus \mathcal{O}_{\mathbb{P}^{1}}^{\oplus q_{-1}}(-1) \bigoplus_{i=0}^{m} \mathcal{O}_{\mathbb{P}^{1}}^{\oplus q_{i}}(i) .
$$

Then

$$
\mathcal{E}=\bigoplus_{i=0}^{m} \mathcal{O}_{\mathbb{P}^{1}}^{\oplus q_{i}}(i)
$$

In particular, if $r_{f}$ is the rank of $\mathcal{E}$, then

$$
r_{f}=\sum_{i=0}^{m} q_{i} .
$$

Theorem A in the introduction is about a relation between $r_{f}$ and the irregularity $q$ when $S$ is a GH-surface. It is a straightforward consequence of the following one. Let $S$ be a surface of general type with a rational pencil $\Lambda$ of irreducible curves. Consider $\sigma: X \rightarrow S$ a minimal composition of blow-ups which resolves the base points of $\Lambda$. In particular, $\Lambda$ induces a relatively minimal fibration $f: X \rightarrow \mathbb{P}^{1}$ and $\mathcal{E}$, the rank- $r_{f}$ subsheaf generically generated by the global sections of $f_{\star} \mathcal{O}\left(K_{X}\right)$, remains defined.

Theorem 3.1. Let $S$ be a minimal surface of general type with a rational pencil $\Lambda$ of irreducible curves. If the Albanese image of $S$ is a curve and $q=r_{f}+1 \geq 2$, then $S$ is $G H$.

Proof. We put $r_{f}=r$. Since $h^{0}(\mathcal{E})=p_{g}$ and $h^{1}(\mathcal{E})=0$, by Riemann-Roch for a vector bundle on a curve we have

$$
\operatorname{deg} \mathcal{E}=p_{g}-r .
$$


On the other hand, by (3), the maximal degree of an $\mathcal{E}$-factor satisfies the inequality $m r \geq \operatorname{deg} \mathcal{E}$; that is,

$$
m \geq \frac{p_{g}-r}{r} .
$$

This means that $K_{X}-m C_{1}$ is effective. Now we use the other hypothesis. The Albanese map of $X$ factors through the Albanese map of $S$, and so we have another fibration $\alpha_{X}: X \rightarrow C \subset \operatorname{Alb}(S)$ over a genus $q$ curve. Obviously the two fibrations $f$ and $\alpha_{X}$ must be transversal, and if $C_{2}$ is the fibre of $\alpha$, for the intersection product we have $\left.\left(K_{X}-m C_{1}\right) C_{2}\right) \geq 0$. By adjunction and (4) we can write

$$
2 g_{2}-2=K_{X} C_{2} \geq m C_{1} C_{2} \geq C_{1} C_{2} \frac{p_{g}-r}{r} .
$$

By hypothesis $X$ is not birational to $\mathbb{P}^{1} \times C$. Therefore $C_{1} C_{2} \geq 2$ and (5) implies

$$
g_{2}-1 \geq \frac{p_{g}-r}{r} \text {. }
$$

We are ready to use the identity $q=r+1$. In fact by Fujita's theorem

$$
\operatorname{deg} \alpha_{\star} \omega_{X \mid C}=\chi\left(\mathcal{O}_{X}\right)-\left(g_{2}-1\right)(q-1) \geq 0,
$$

and by (6) we obtain

$$
p_{g}-r=\chi\left(\mathcal{O}_{X}\right) \geq\left(g_{2}-1\right)(q-1) \geq \frac{p_{g}-r}{r}(q-1)=p_{g}-r .
$$

In particular, it follows that $0=\operatorname{deg} \alpha_{\star} \omega_{X \mid C}=\operatorname{deg} \alpha_{\star} \omega_{S \mid C}$. Now by [2.3, $S$ is $\mathrm{GH}$.

In the following propositions we show the converse of Theorem 3.1 under the extra assumption that the condition $q(S)=r_{f}+1$ holds for the natural fibration $f: S \rightarrow \mathbb{P}^{1}=C_{2} / G$ of a GH-surface. First we analyze the case with $G=\mathbb{Z} / \mathbb{Z}_{2}$.

Proposition 3.2. If $S=C_{1} \times C_{2} / G$ is a GH-surface and $G=\mathbb{Z} / \mathbb{Z}_{2}$, then $q(S)=$ $r_{f}+1$.

Proof. By hypothesis $C_{1} \rightarrow C_{1} / G=C$ is unramified, and from Riemann-Hurwitz it follows that $g_{1}=1+2(q-1)$. On the other hand, (3) says that $g_{1}=q+q_{-1}+r_{f}$; then

$$
q+q_{-1}+r_{f}=1+2(q-1) .
$$

Now we have to recall the following inequality, proved in $[\mathrm{K}$. Proposition 1.5]: $r_{f} \geq q-1$. This together with (7) leads to the claim, since we have $q_{-1}=0$ and $q(S)=r_{f}+1$.

We want to stress a subtle point. It can happen that on a surface we have a fibration $\phi: X \rightarrow \mathbb{P}^{1}$ with $q(X)=r_{\phi}+1$. Thus its minimal model $S=C_{1} \times C_{2} / G$ is a GH-surface, but the natural pencil $f: S \rightarrow \mathbb{P}^{1}=C_{2} / G$ does not share anything with $\phi$.

Remark 3.3. Surfaces with $p_{g}=q=2$ provide a large class of GH-surfaces $S=$ $C_{1} \times C_{2} / G$ such that the induced canonical pencil (over a suitable blow-up) satisfies the condition $q(S)=r_{\phi_{|K|}}+1$, but $G \neq \mathbb{Z}_{2}$. In fact, the canonical fibration for these surfaces is not the natural fibration $f: S \rightarrow \mathbb{P}^{1}=C_{2} / G$. See Proposition 5.3 
We are now ready to appreciate the converse of 3.2 From the introduction we recall that if $G^{\star}$ is the set of the linear characters of $G$, then the induced representations on $H^{0}\left(\Omega_{C_{j}}^{1}\right), j=1,2$, give the decompositions $H^{0}\left(\Omega_{C_{1}}^{1}\right)=\bigoplus_{\chi \in G^{\star}} V^{\chi}$, $H^{0}\left(\Omega_{C_{2}}^{1}\right)=\bigoplus_{\chi \in G^{\star}} W^{\chi}$ where $G$ acts on $V^{\chi}, W^{\chi}$ via $\chi$. By the same proof of [Z] Theorem 1.4], we have

$$
H^{0}\left(S, \omega_{S}\right)=\bigoplus_{\chi \in G^{\star}} V^{\chi} \otimes W^{\chi^{\vee}}
$$

$\chi^{\vee}$ being the dual representation.

Proposition 3.4. Let $S=C_{1} \times C_{2} / G$ be a GH-surface such that $q=r_{f}+1$ where $f: S \rightarrow \mathbb{P}^{1}=C_{2} / G$ is the natural fibration with generic fibre isomorphic to $C_{1}$. Then $G=\mathbb{Z}_{2}$.

Proof. Since $\pi_{1}: C_{1} \rightarrow C=C_{1} / G$ is unramified, it is easy to prove that for every $\chi \in G^{\star}$ we have $\operatorname{dim}_{\mathbb{C}} V^{\chi} \geq q-1$ where $q=g(C)>1$. Let

$$
I=\left\{\chi \in G^{\star} \mid W^{\chi^{\vee}} \neq 0\right\} \text {; }
$$

then

$$
r_{f}=\sum_{\chi \in I} \operatorname{dim}_{\mathbb{C}} V^{\chi}
$$

By (8) it follows that there exists a unique $\chi \in G^{\star}$ such that $W^{\chi^{\vee}} \neq 0$. This is also true for all the morphisms $C_{2} \rightarrow C_{2} / H$ where $H<G$. To prove our statement it remains to prove

Claim. Let $C, D$ be two smooth curves and $H \neq \mathbb{Z}_{2}$ an abelian group acting faithfully on $C$ with $D=C / H$. Then there exist at least two nontrivial characters $\chi_{1}, \chi_{2} \in G^{\star}$ such that $V^{\chi_{1}} \neq 0, V^{X_{2}} \neq 0$.

Proof of the claim. We can easily assume first that $H$ is cyclic, then that the character $\chi$ is such that $H^{0}\left(C, \omega_{C}\right)=H^{0}\left(D, \omega_{D}\right) \oplus V^{X}$ is a generator of the group of characters. In particular, the action over the ramification loci (cf. [Pa, Lemma 1.2]) is given only via $\chi$ : a contradiction by $[\mathrm{Pa}$, Formula 2.15].

\section{Finite GRoups}

We will show that for every $n, m>1$ there exists a GH-surface with Albanese fibration of genus $n$ and irregularity $m$. First we will show that for every nontrivial group $G$ there exists an infinite family $\left\{S_{n}\right\}_{n \in \mathbb{N}}$ (i.e., with $\lim _{n \rightarrow \infty} \chi\left(S_{n}\right)=\infty$ ) of GH-surfaces with fibre of the Albanese map of genus $g$.

Lemma 4.1. Let $G$ be a nontrivial finite group. Then there exists an algebraic curve $C_{2}$ such that $g_{2} \geq 2, G \subset \operatorname{Aut}\left(C_{2}\right)$ and $\mathbb{P}^{1}=C_{2} / G$.

Proof. This is a well-known result by Hurwitz. See, for example, $\mathrm{Br}$, Corollary 3.15].

Lemma 4.2. Let $G$ be finite group. Then there exist two (connected) curves $C$ and $D$, both with genus $\geq 2$, such that $C$ is a $G$-Galois unramified cover of $D$.

Proof. If $G$ is cyclic the proof is easy; otherwise we fix a finite noncyclic group $G$ and let $\left\{x_{1}, \ldots, x_{g}\right\}, g \geq 2$, be its generators. The fundamental group of $D$ is $\Pi_{1}(D)=\left\langle a_{1}, b_{1}, \ldots, a_{g}, b_{g} \mid\left[a_{1}, b_{1}\right] \ldots\left[a_{g}, b_{g}\right]=1\right\rangle$, and over the generators we define $a_{i}, b_{i} \stackrel{\phi}{\mapsto} x_{i}$, which extends to a homomorphism $\phi: \Pi_{1}(D) \rightarrow G$. Let $\Pi_{1}$ be the 
kernel of $\phi$. Then $\Pi_{1}$ is a normal subgroup and $G=\Pi_{1}(D) / \Pi_{1}$ acts on the Riemann surface $C=\mathbb{H} / \Pi_{1}$ where $\mathbb{H}$ is the hyperbolic plane and $\Pi_{1}=\Pi_{1}(C)$.

Lemmas 4.1 and 4.2 easily give the following:

Proposition 4.3. For every finite nontrivial group $G$ there exists a $G H$-surface $S$ such that $S=C_{1} \times C_{2} / G$.

Proof. Fix a finite nontrivial group $G$. Then by 4.1 there exists an algebraic curve $C_{2}$ such that $g_{2} \geq 2$ and $\mathbb{P}^{1}=C_{2} / G$. By 4.2 there exist $C_{1}$ and $C$ such that $C_{1}$ is a $G$-Galois unramified cover of $C$. Then there remains defined a diagonal action of $G$ onto $X=C_{1} \times C_{2}$ such that $\mathrm{S}=X / G$ is a GH-surface.

Once we have constructed a GH-surface $S=C_{1} \times C_{2} / G$, by the base change over its Albanese morphism we can produce an infinite family $\left\{S_{n}\right\}_{n \in \mathbb{N}}$ of GH-surfaces, and it is now easy to show the following:

Proposition 4.4. For every finite nontrivial group $G$, there exists an integer $g \gg 0$ (actually infinitely many) and an infinite family of $G H$ surfaces with group $G$ and with Albanese fibration of genus $g$.

Proof. Trivial by 4.3

Proposition 4.5. For every $n, m>1$ there exists a GH surface with Albanese fibration of genus $n$ and irregularity $m$.

Proof. Let $C_{2}$ be a hyperelliptic curve of genus $g_{2}=n$. Let $G=\mathbb{Z} / 2$ be the group generated by the involution. Fix a generic curve $C$ of genus $m>1$. Then there exists a $G$-unramified cover $C_{1} \rightarrow C$. Finally, the natural map $C_{1} \times C_{2} / G \rightarrow C$ gives a fiber bundle structure of genus $n$ over a GH-surface with irregularity $m$.

These results are closely related to the geometry of hyperelliptic curves (cf. $\mathrm{Ca}$ Corollary 3.20]), and they are among the first consequences one can obtain by Galois theory applied to GH. In the rest of the paper $G$ will be a finite group, not necessarily a commutative one.

\section{Theorem B}

We classify the GH-surfaces with canonical map composed with a pencil, and in doing so we give a complete answer to $[\mathrm{K}$, Theorem 3.6]. The following proposition is the first claim of Theorem B in the introduction.

Proposition 5.1. Let $S$ be a surface of general type with canonical map composed with a pencil $\Lambda$, and let $q=2$. If its Albanese image is a curve, then $S$ is $G H$.

Proof. By $[\mathrm{X}], \Lambda$ is a rational pencil, and if $\lambda: S \rightarrow \mathbb{P}^{1}$ is the rational map induced by $\Lambda$, the fibres are connected. We consider $\epsilon: Y \rightarrow S$ a minimal composition of blow-ups to resolve the base points, and we call $\psi=\lambda \circ \epsilon$ the induced fibration. Let $F$ be a general fiber; then $\mathcal{O}_{Y}(F)_{\mid F}=\mathcal{O}_{F}$. In particular, we can write $\mathcal{O}_{F}\left(K_{F}\right)=$ $\mathcal{O}_{Y}\left(K_{Y}+F\right)_{\mid F}=\mathcal{O}_{Y}\left(K_{Y}\right)_{\mid F}$. Then we have a restriction morphism on the spaces of global forms: $r_{F}: H^{0}\left(Y, \mathcal{O}_{Y}\left(K_{Y}\right)\right) \rightarrow H^{0}\left(F, \mathcal{O}_{F}\left(K_{F}\right)\right)$. Since the canonical map of $Y$ is composed with $\psi$, then $\operatorname{dim} r_{F}\left(H^{0}\left(Y, \mathcal{O}_{Y}\left(K_{Y}\right)\right)\right)=1$, for every $F$. This means that the subvector bundle $\mathcal{E}$ of $\psi_{\star} \omega_{Y}$ generically generated by the global sections has rank $r$ equal to 1 ; now the claim follows by 3.1 
For further reference we set aside the following:

Remark 5.2. If $S$ is a $G H$-surface with canonical map composed with a pencil $\Lambda$, then $\phi_{\Lambda}: S \rightarrow \mathbb{P}^{1}$ has generically irreducible fiber. Moreover, $\phi_{\Lambda}$ is said to be the canonical pencil.

Since we are going to classify all GH-surfaces with canonical map composed with a pencil, the above proposition says that we actually classify all surfaces with $q=2$, not of Albanese general type and with canonical map composed with a pencil.

Proposition 5.3. Let $S$ be a GH-surface with canonical map composed with a pencil. Then either $p_{g}=q=2$, or it is a $G=\mathbb{Z} / 2$ quotient of $X=C_{1} \times C_{2}$ where $C_{1}$ is a genus-3 curve and $G$ acts freely on it.

Proof. Let $\lambda: S \rightarrow B$ be the fibration induced by the global sections of the canonical sheaf. In 5.1 we recalled that $B=\mathbb{P}^{1}$. Now by definition

$$
\left|K_{S}\right| \equiv Z+\left|\left(p_{g}-1\right) F\right|
$$

where $F$ is the fiber of $\lambda, Z$ is the fixed component of $\left|K_{S}\right|$, and $\equiv$ means "linearly equivalent". Inside $Z$ we distinguish two components: $Z=H+V$ where $V$ is supported over all irreducible components of $Z$ contained in the fibers of $\lambda$ while $H$ is supported over the irreducible components with positive intersection with $F$; in particular, $H F=2 g(F)-2$. Since $S$ is $\mathrm{GH}$, then $q>1$, and by $[\mathrm{X}$ we have $q=2$. In particular, if $p_{g}=2$, we have the claim. Assume that $p_{g}>2$. For the reader's benefit we stress that $f: S \rightarrow \mathbb{P}^{1}$ is one of the two fibrations naturally associated to a GH-surface. First we show that $\lambda=f$, by contradiction. Assume that $\lambda \neq f$. We cannot use the condition $r=1$ because $f \neq \lambda$ ! On the other hand, the images of $C_{1}$ and $C_{2}$ are equal to the canonical image $\Sigma$, which is a curve in $\mathbb{P}^{p_{g}-1}$. Then, since $q=2$, by (2) we have $g_{1}=p_{g}+q_{-1}+2$ and $g_{2}=p_{g}$. We apply the adjunction formula twice:

$$
\begin{gathered}
2\left(p_{g}+q_{-1}+2\right)-2=Z C_{1}+\left(p_{g}-1\right) F C_{1}, \\
2 p_{g}-2=Z C_{2}+\left(p_{g}-1\right) F C_{2} .
\end{gathered}
$$

By (10), $Z C_{2}=0$ and $C_{2} F=2$. Then $Z$ is a union of components in the fibres of the holomorphic bundle $\alpha: S \rightarrow C$. By the projection formula and the RiemannRoch theorem on $C$ we have $1=h^{0}\left(S, \mathcal{O}_{S}(Z)\right)=h^{0}\left(C, f_{\star} \mathcal{O}_{S}(Z)\right) \geq d-1$ where $d=\operatorname{deg}\left(f_{\star} \mathcal{O}_{C}(Z)\right)$; that is,

$$
d \leq 2 \text {. }
$$

If $d=1$, then $Z F=2$. In particular, $g(F)=2$ : a contradiction with $[\mathrm{X}]$. If $d=2$, then $Z C_{1}=2 C_{2} C_{1}=2|G|$. On the other hand, the Riemann-Hurwitz formula for the unramified morphism $C_{1} \rightarrow C=C_{1} / G$ says that $2|G|=2\left(p_{g}+q_{-1}+2\right)-2$. Then by (9) $F C_{1}=0$; that is, $\lambda=f$.

Now we assume $\lambda=f$. In particular, since $q=2, r=1$, by (2) the genus of the fibration induced by the canonical map is

$$
g_{1}=2+q_{-1}+1 \text {. }
$$

We are going to show that in this case $q_{-1}=0$. Once we have proved $q_{-1}=0$, then $g_{1}=3$, and the claim will follow immediately since $C_{1}$ must be an uramified cover of the image of the Albanese map, which is a genus- 2 curve.

Since $g_{2}=p_{g}$ and $r=1$, then by (5) in Theorem 3.1 we have $C_{1} C_{2}=2$. Then $G=\mathbb{Z} / 2$. By the proof of 3.2 we have $q_{-1}=0$. 
Let $S$ be a GH-surface with $p_{g}=q=2$ and, as above, consider $\epsilon: Y \rightarrow S$ a minimal composition of blow-ups to resolve the base points; call $\psi=\lambda \circ \epsilon$ the induced fibration. Assume that the fibres of the canonical pencil have genus $h>3$. Then, by [ $\mathrm{K}$ Theorem 3.6], in order to finish the proof of Theorem B we only need to exclude the case $h=6$. Notice that if $\left|K_{S}\right|$ has no fixed part, then $h=1+K_{S}^{2}=9$.

Lemma 5.4. Let $S$ be a GH-surface with $p_{g}=q=2$ and with canonical map composed with a pencil of genus $>3$. Then the general element of $\left|K_{S}\right|$ is a smooth curve of genus 9 .

Proof. Let us consider the orbits map $C_{1} \times C_{2}=X \stackrel{\pi}{\rightarrow} S=C_{1} \times C_{2} / G$. Let $\operatorname{tr}: H^{0}\left(X, \omega_{X}\right) \rightarrow H^{0}\left(S, \omega_{S}\right)$ be the trace homomorphism; then $\pi^{\star} \circ \operatorname{tr}$ is the multiplication by $|G|$. Since $p_{g}=2$, the invariant subspace $V=H^{0}\left(X, \omega_{X}\right)^{G}$ is 2-dimensional. Since the canonical morphism is not composed with the Albanese pencil $\alpha: S \rightarrow C$, which is a genus 2 pencil with fiber $C_{2}$, it follows that the restriction homomorphism $V \rightarrow H^{0}\left(C_{2}, \omega_{C_{2}}\right)$ is an isomorphism. It follows that $V=\left\langle\omega_{1} \wedge \eta_{1}, \omega_{2} \wedge \eta_{2}\right\rangle$ where $\left\langle\omega_{1}, \omega_{2}\right\rangle=H^{0}\left(\Omega_{C_{2}}^{1}\right)$ and $\left\langle\eta_{1}, \eta_{2}\right\rangle \subset H^{0}\left(\Omega_{C_{1}}^{1}\right)$. Since there do not exist geometrical fibres of $\alpha$ in the fixed loci of $\left|K_{S}\right|$, then $|V|$ has only simple base points, and by Bertini it follows that the general element $H \in \Lambda$ is smooth. Hence it is irreducible, because each of its components is numerically equivalent to a sum of fibres of the natural projections $X=C_{i} \times C_{2} \rightarrow C_{i}, i=$ 1,2 . Obviously $G$ acts over $H$, since $H$ is the zero scheme of an invariant 2 -form. Moreover, $H / G$ is smooth, because $G$ has no fixed points. Since $\pi: X \rightarrow S$ is a $G$-Galois unramified cover, it follows by the very nature of the trace morphism that $H / G \in\left|K_{S}\right|$. In particular, $g(H / G)=9$.

Theorem B in the introduction is the content of 5.15 .3 and 5.4

\section{Theorem C}

In the previous section we have classified the GH-surfaces with $r=1$. In this section we classify those with $r=2$; that is, the GH-surfaces such that the canonical image is a ruled surface where the fibers of the ruling are immersed as lines. In fact, the condition $r=2$ means that $\phi_{\left|K_{S}\right|}\left(C_{1}\right)=\mathbb{P}^{1} \subset \mathbb{P}^{p_{g}-1}$.

Example: $r=2, q=3$. Denote by $C_{1}$ a curve of genus 5 with a free $G=\mathbb{Z} / 2$ action and let $C=C_{1} / G$ be the genus-3 quotient. Then we have the following decomposition:

$$
H^{0}\left(C_{1}, \omega_{C_{1}}\right)=V^{+} \oplus V^{-},
$$

where $V^{+}=H^{0}\left(C, \omega_{C}\right)$ is 3-dimensional. Consider any hyperelliptic curve $C_{2}$ with its group $G=\langle j\rangle$ generated by the hyperelliptic involution $j$; in a self-explanatory notation we can set $H^{0}\left(C_{2}, \omega_{C_{2}}\right)=W^{-}$. In particular, if $S=C_{1} \times C_{2} / G$, then

$$
H^{0}\left(S, \omega_{S}\right)=V^{-} \otimes W^{-},
$$

and we have $r=2, q=3$.

Proposition 6.1. Let $S$ be a GH-surface with $r=2$. If $q \geq 3$, then $S$ is one of the surfaces constructed in the example; in particular, $q=3$. Otherwise $q=2$ and $3 \leq|G| \leq 6$. 
Proof. First step. Assume $|G| \geq 3$. We notice that in $S$ the intersection number $C_{1} C_{2}=|G|$. From the inequality (5) in the proof of 3.1 we have $2 g_{2}-2 \geq \frac{3}{2}\left(p_{g}-2\right)$, i.e., $g_{2} \geq \frac{3}{4} p_{g}-\frac{1}{2}$. Since $\chi\left(\mathcal{O}_{X}\right) \geq\left(g_{2}-1\right)(q-1)$, then $\chi\left(\mathcal{O}_{X}\right) \geq\left(\frac{3}{4} p_{g}-\frac{3}{2}\right)(q-1)$, and this implies

$$
p_{g} \geq\left(\frac{3}{4} p_{g}-\frac{1}{2}\right)(q-1) .
$$

Now if $q \geq 4$, then $\frac{12}{10} \geq p_{g}$, and if $q=3$, then $2 \geq p_{g}$ : in both cases we have a contradiction. We have shown that $|G| \geq 3$ implies $q=2$.

Second step. Assume $G=\mathbb{Z} / 2$. We have

$$
p_{g} \geq p_{g} \frac{q-1}{2} .
$$

Then $q \leq 3$. If $q=3$, we have the claim. If $q=2$, we still have $H^{0}\left(S, \omega_{S}\right)=V^{-} \otimes$ $W^{-}$as in the example, but this time $C_{1}$ has genus 3 ; hence $V^{-}$is 1-dimensional, that is, $r=1$.

Third step. Assume $q=2$; then $p_{g}=g_{2}$ and to show that $3 \leq|G| \leq 6$ we consider two cases: $p_{g}=3$ or $p_{g}>3$. If $p_{g}=3$, then in (5) we have $m=1$, which implies $|G|=4$; in the other case, from (5) we have $4 p_{g}-4 \geq|G|\left(p_{g}-2\right)$, that is, $2|G|-4 \geq(|G|-4) p_{g} \geq 4(|G|-4)$, since $p_{g} \geq 4$. This implies $|G| \leq 6$.

Remark 6.2. To show that $r=2$ implies $q \leq 3$, one can also use [K] Lemma 1.6].

Now we are ready to give a complete classification of GH-surfaces whose canonical image is a ruled surface immersed in $\mathbb{P}^{p_{g}-1}$ by sections of the fibration induced by $f$. We remark that in the proof of Theorem $\mathrm{C}$ we will construct each occurring class explicitly.

Proof of Theorem C. Following the proof of 6.1, we see that case described in the example. If $q=2$, then $3 \leq|G| \leq 6$. Moreover, if $G=\mathbb{Z} / 5$, then $3 \leq p_{g} \leq 6$; if $G=\mathbb{Z} / 2 \times \mathbb{Z} / 3$ or $G=\mathcal{S}_{3}$, then $3 \leq p_{g} \leq 4$. Now the proof splits into 7 cases. In every case let $H^{0}\left(C_{i}, \omega_{C_{i}}\right)=\bigoplus_{\chi \in G \star} V_{i}^{\chi}$ be the decomposition of the space of global 1-forms into the direct sum of the subspaces $V_{i}^{\chi}$ where $G$ acts via the character $\chi$ (and where now we have not put $i=1,2$ inside parentheses as in the introduction).

The global $G$-invariant 2-forms of $X=C_{1} \times C_{2}$ give the global 2-forms on $S$, and by $[\mathbf{Z}$, Theorem 1.4] we have

$$
H^{0}\left(S, \omega_{S}\right)=\bigoplus_{\chi \in G^{\star}} V_{1}^{\chi} \otimes V_{2}^{\chi^{-1}}
$$

Then $r=2$ if and only if one of the two following cases occurs: $(i)$ there exists a unique $V_{1}^{\chi}$ such that $\operatorname{dim}_{\mathbb{C}} V_{1}^{\chi}=2, \operatorname{dim}_{\mathbb{C}} V_{1}^{\chi} \otimes V_{2}^{\chi^{-1}} \geq 3$ and $V_{1}^{\tilde{\chi}} \otimes V_{2}^{\tilde{\chi}^{-1}}=0$ for every $\tilde{\chi} \neq \chi$, or $(i i)$ there exist $V_{1}^{\chi_{j}}$ such that $\operatorname{dim}_{\mathbb{C}} V_{1}^{\chi_{j}}=1, j=1,2, V_{1}^{\chi_{j}} \otimes V_{2}^{\chi_{j}^{-1}} \neq 0$, $\operatorname{dim}_{\mathbb{C}} \bigoplus_{j=1}^{2} V_{1}^{\chi_{j}} \otimes V_{2}^{\chi_{j}^{-1}} \geq 3$ and $V_{1}^{\chi} \otimes V_{2}^{\chi^{-1}}=0$ for every $\chi \neq \chi_{1}$ or $\chi \neq \chi_{2}$. We will recall some details of the covering theory in the course of the proof.

First we consider the case

$$
G=\mathbb{Z} / 3 .
$$

The $\mathbb{Z} / 3$-unramified covering $\pi_{1}: C_{1} \rightarrow C$ can be reconstructed by an element $\mathcal{M}_{\chi} \in \operatorname{Pic}_{3}^{0}(C)$ such that $\pi_{1, \star} \mathcal{O}_{C_{1}}=\mathcal{O}_{C} \oplus \mathcal{M}_{\chi}^{\vee} \oplus \mathcal{M}_{\chi^{2}}^{\vee}$ cf. [Pa, Formula 1.1]. Moreover,

$$
H^{0}\left(C_{1}, \omega_{C_{1}}\right)^{\chi^{i}}==H^{0}\left(C, \omega_{C} \otimes \mathcal{M}_{\chi^{-i}}\right), \quad i=1,2 .
$$


Since $H^{1}\left(C, \omega_{C} \otimes \mathcal{M}_{\chi^{i}}\right)=0$, by Riemann-Roch over $C$ we have $\operatorname{dim}_{\mathbb{C}} V_{1}^{\chi^{i}}=1$, where $i=1,2$. This implies, without loss of generality, that the other cover, $\pi_{2}: C_{2} \rightarrow \mathbb{P}^{1}$, must satisfy the two conditions $\operatorname{dim}_{\mathbb{C}} V_{2}^{\chi}>1, \operatorname{dim}_{\mathbb{C}} V_{2}^{\chi^{2}}>1$. We point out all the details in this case, so that the similar computations can be omitted in the rest of the proof. The covering $\pi_{2}: C_{2} \rightarrow \mathbb{P}^{1}$ is given by two line bundles $\mathcal{L}_{\chi}, \mathcal{L}_{\chi^{2}}$ on $C$, and two effective divisors $D_{1}, D_{2}$ such that the branch loci is $D=D_{1}+D_{2}$ and in $\operatorname{Pic}\left(\mathbb{P}^{1}\right) \simeq \mathbb{Z}$ we have

$$
\left\{\begin{array}{l}
3 \mathcal{L}_{\chi}=D_{1}+2 D_{2} \\
\mathcal{L}_{\chi^{2}}=2 \mathcal{L}_{\chi}-D_{2}
\end{array}\right.
$$

The conditions $\operatorname{dim}_{\mathbb{C}} V_{2}^{\chi}>1, \operatorname{dim}_{\mathbb{C}} V_{2}^{\chi^{2}}>1, \operatorname{dim}_{\mathbb{C}} \bigoplus_{j=1}^{2} V_{1}^{\chi_{j}} \otimes V_{2}^{\chi_{j}^{-1}} \geq 3$ imply, respectively, the following ones: $l_{1}=\operatorname{deg} \mathcal{L}_{\chi} \geq 2, l_{2}=\operatorname{deg} \mathcal{L}_{\chi^{2}} \geq 2$ and $l_{1}+l_{2} \geq 5$. If we set $\operatorname{deg} D_{i}=d_{i}$, where $i=1,2$, then by (12) and the Hurwitz formula we can write

$$
\left\{\begin{array}{l}
2 g_{2}-2=-6+2 d_{1}+2 d_{2}, \\
6 \leq 3 l_{1}=d_{1}+2 d_{2}, \\
4 \leq 2 l_{1}=l_{2}+d_{2}, \\
l_{1}+l_{2} \geq 5
\end{array}\right.
$$

The solution is easily found as the set of numbers $\left\{l_{1}, l_{2}, d_{1}, d_{2}, g_{2}\right\}$ such that $l_{1} \geq 2$, $l_{2} \geq 2, l_{1}+l_{2} \geq 5$ and

$$
\left\{\begin{array}{l}
g_{2}+2=l_{1}+l_{2}, \\
d_{1}+d_{2}=l_{1}+l_{2}, \\
2 l_{1}=d_{2}+l_{2} .
\end{array}\right.
$$

Now it is not difficult to see that for every $g_{2}=m \geq 3$ there are $\frac{m+2}{2}$ or $\frac{m+1}{2}$ classes of surfaces $S$ such that $S=C_{1} \times C_{2} / \mathbb{Z} / 3$ with $g_{1}=4, p_{g}=g_{2}=m, q=2$ and the canonical image is a ruled surface whose elements of the ruling are immersed as lines in $\mathbb{P}^{m-1}$.

The next case is

$$
G=\mathbb{Z} / 4 \text {. }
$$

The covering is given by a primitive line bundle $\mathcal{M}_{\chi} \in \operatorname{Pic}_{4}^{0}$; then $\operatorname{dim}_{\mathbb{C}} V_{1}^{\chi^{i}}=1$, where $i=1,2,3$.

This implies that the other cover must satisfy the condition $\operatorname{dim}_{\mathbb{C}} V_{2}^{\chi^{i}}=0$ for exactly one among the $i$ 's. In particular, $\pi_{2}: C_{2} \rightarrow \mathbb{P}^{1}$ is given by three line bundles $\mathcal{L}_{\chi}, \mathcal{L}_{\chi^{2}}, \mathcal{L}_{\chi^{3}}$ on $C$ and three effective divisors $D_{1}, D_{2}$ and $D_{3}$ such that

$$
\left\{\begin{array}{l}
4 \mathcal{L}_{\chi}=D_{1}+2 D_{2}+3 D_{3}, \\
\mathcal{L}_{\chi^{2}}=2 \mathcal{L}_{\chi}-D_{2}-D_{3}, \\
\mathcal{L}_{\chi^{3}}=3 \mathcal{L}_{\chi}-D_{2}-2 D_{3}
\end{array}\right.
$$

If we set $\mathcal{L}_{\chi}=1, \operatorname{deg} \mathcal{L}_{\chi^{2}}=l_{2}, \operatorname{deg} \mathcal{L}_{\chi^{3}}=l_{3}, \operatorname{deg} D_{i}=d_{i}, i=1,2,3$, and take the degrees, we obtain from (15) the numerical constraints

$$
\left\{\begin{array}{l}
4=d_{1}+2 d_{2}+3 d_{3}, \\
l_{2}=2-d_{2}-d_{3} \\
l_{3}=3-d_{2}-2 d_{3} .
\end{array}\right.
$$


Since $p_{g} \geq 3$, there exists the unique solution $d_{2}=d_{3}=0, d_{1}=4, l_{2}=2, l_{3}=4$. Then, given an unramified $\mathbb{Z} / 4$-cover of a genus 2 fixed curve $C$ and the unique $\mathbb{Z} / 4$ - covering of $\mathbb{P}^{1}$ branched over four fixed points $D_{1}=P_{1}+P_{2}+P_{3}+P_{4}$, there exists a unique GH-surface with group $G=\mathbb{Z} / 4, g_{2}=p_{g}=3, q=2$. On the other hand, without loss of generality we can assume $l_{2}=1$; then there are two infinite families of solutions:

$(I)_{\mathbb{Z} / 4} d_{1}=0, d_{2}=2 l-2 \geq 4, d_{3}=2, l_{1}=l, l_{2}=1, l_{3}=l-1 \geq 4 ;$

$(I I)_{\mathbb{Z} / 4} d_{1}=1, d_{2}=2 l-2 \geq 4, d_{3}=1, l_{1}=l, l_{2}=1, l_{3}=l \geq 3$.

Our third case is

$$
G=\mathbb{Z} / 2 \times \mathbb{Z} / 2 .
$$

The data for $\pi_{1}: C_{1} \rightarrow C$ are three nontrivial 2-torsion elements in $\mathrm{Pic}_{2}^{0}$. Then all the $V_{1}^{\chi}$ are 1-dimensional. In this case we can build $S$ if and only if we find three line bundles $\mathcal{L}_{\chi_{i}}, i=1,2,3$, on $\mathbb{P}^{1}$ and three effective divisors $D_{i}, i=1,2,3$, respectively of degree $\operatorname{deg} \mathcal{L}_{\chi_{i}}=l_{i}, \operatorname{deg} D_{i}=d_{i}$ such that, without loss of generality, $l_{1}=1$ and

$$
\left\{\begin{array}{l}
2=d_{1}+d_{3}, \\
2 l_{2}=d_{2}+d_{3}, \\
l_{3}=1+l_{2}-d_{3} .
\end{array}\right.
$$

We easily have two different infinite families of such surfaces:

$(I)_{\mathbb{Z} / 2 \times \mathbb{Z} / 2} d_{1}=2, d_{2}=2 l \geq 4, d_{3}=0, l_{1}=1, l_{2}=l \geq 2, l_{3}=l+1 \geq 4 ;$

$(I I)_{\mathbb{Z} / 2 \times \mathbb{Z} / 2} d_{1}=2, d_{2}=2 l-1 \geq 4, d_{3}=1, l_{1}=1, l_{2}=l_{3}=l \geq 3$.

By the same technique, although with more computations, we can show that the cases $G=\mathbb{Z} / 5, G=\mathbb{Z} / 6$ do not occur. Here we will only show how the case $G=\mathcal{S}_{3}$ can be excluded. One can use the full theory of dihedral coverings as developed in [To]. We give a more direct proof.

So, our last case is

$$
G=\mathcal{S}_{3} \text {. }
$$

If $g_{2}=3$, we exclude the existence of a Galois cover $C_{2} \rightarrow \mathbb{P}^{1}$ with Galois group the symmetric group on three elements by the Hurwitz formula and the following remark.

Claim. Let $\pi: D \rightarrow \mathbb{P}^{1}$ be a connected Galois covering with Galois group the symmetric group over three elements. Let $s=a+b$ be the number of points in the branch loci $\mathcal{B}$ of $\pi$, where $a$ is the number of those with index 2 and $b$ with index 3. Then $a$ is an even number and $b \equiv 0 \bmod (3)$.

In fact, if $\pi$ is given, then we have the orbifold exact sequence of $[\mathrm{Ca}$, Definition 4.4]. In this case we can write $N=(2, \ldots, 2 ; 3, \ldots, 3)$, and $\Pi_{1}^{\text {orb }}(B \backslash \mathcal{B} \mid N)$ is the group

$$
\left\langle\gamma_{1}, \ldots, \gamma_{a}, \mu_{1}, \ldots, \mu_{b} \mid \gamma_{1} \ldots \gamma_{s} \mu_{1} \ldots \mu_{b}=\gamma_{1}^{2}=\ldots=\gamma_{a}^{2}=\mu_{1}^{3}=\ldots=m u_{b}^{3}=1\right\rangle .
$$

Since $G=\left\langle x, y \mid a^{2}=b^{3}=1, a b a=b^{-1}\right\rangle$, then the condition of the orbifold exact sequence implies $\gamma_{i} \mapsto a$, and $\mu_{j} \mapsto b$ or $b^{-1}$, where $i=1, \ldots, a$ and $j=1, \ldots, b$. The claim follows, since we can similarly verify the other condition, $\gamma_{1} \ldots \gamma_{s} \mu_{1} \ldots \mu_{b}=1$ and $D$ is connected.

We turn to the main point. We cannot exclude the case where $C_{2} \rightarrow \mathbb{P}^{1}$ is an $\mathcal{S}_{3}$-covering where $C_{2}$ has genus $g_{2}=4, s=5, a=2$ and $b=3$ : this covering does exist by the above claim and the orbifold exact sequence. By the same reason the $\mathcal{S}_{3}$-unbranched covering $C_{1} \rightarrow C$ also exists where $C$ is a genus-2 curve. So 
a GH-surface with the "right" invariants does exist, but $r=3$. Let us show that $r=3$.

We know from representation theory, or by hand, that $\mathcal{S}_{3}$ has three irreducible characters which correspond to the three possible type of irreducible subspaces. Let $H=\langle a\rangle \simeq \mathbb{Z} / 2$.

Since $C_{1}$ has genus 7 and $C_{1} / H$ has genus 4 , among all the possible decompositions of the 7-dimensional space $H^{0}\left(C_{1}, \mathcal{O}_{C_{1}}\right)$ into irreducible subspaces we have to consider only the following one:

$$
H^{0}\left(\Omega_{C_{1}}^{1}\right)=H^{0}\left(\Omega_{C}^{1}\right) \oplus U \oplus V \oplus W
$$

where $U=\left\langle u_{1}, u_{2}\right\rangle, V=\left\langle v_{1}, v_{2}\right\rangle, W=\langle w\rangle$ and $\mathcal{S}_{3}$ acts in the following way:

$$
a:\left\{\begin{array}{l}
u_{1} \mapsto u_{2}, \\
u_{2} \mapsto u_{1}, \\
v_{1} \mapsto v_{2}, \\
v_{2} \mapsto v_{1}, \\
w \mapsto-w,
\end{array} \quad b:\left\{\begin{array}{l}
u_{1} \mapsto e^{2 \pi i / 3} u_{1}, \\
u_{2} \mapsto e^{4 \pi i / 3} u_{2}, \\
v_{1} \mapsto e^{2 \pi i / 3} v_{1}, \\
v_{2} \mapsto e^{4 \pi i / 3} v_{2}, \\
w \mapsto w .
\end{array}\right.\right.
$$

Notice that $H^{0}\left(\Omega_{C_{1} / H}^{1}\right)=H^{0}\left(\Omega_{C}^{1}\right) \oplus\left\langle u_{1}+u_{2}, v_{1}+v_{2}\right\rangle$. By an analogous argument there is only "one" possible $\mathcal{S}_{3}$-covering $C_{2} \rightarrow \mathbb{P}^{1}$ with $s=5, a=2$ and $b=3$ :

$$
H^{0}\left(\Omega_{C_{2}}^{1}\right)=U^{\prime} \oplus W^{\prime}
$$

where $U^{\prime}=\left\langle u_{1}^{\prime}, u_{2}^{\prime}\right\rangle, W^{\prime}=\left\langle w^{\prime}, w^{\prime \prime}\right\rangle$ and

$$
a:\left\{\begin{array}{l}
u_{1}^{\prime} \mapsto u_{2}^{\prime}, \\
u_{2}^{\prime} \mapsto u_{1}^{\prime}, \\
w^{\prime} \mapsto-w^{\prime}, \\
w^{\prime \prime} \mapsto-w^{\prime \prime},
\end{array} \quad b:\left\{\begin{array}{l}
u^{\prime} \mapsto e^{2 \pi i / 3} u^{\prime}, \\
u^{\prime \prime} \mapsto e^{4 \pi i / 3} u^{\prime \prime}, \\
w^{\prime} \mapsto w^{\prime}, \\
w^{\prime \prime} \mapsto w^{\prime \prime} .
\end{array}\right.\right.
$$

Then

$$
H^{0}\left(S, \omega_{S}\right)=\left\langle w \wedge w^{\prime}, w \wedge w^{\prime \prime}, u_{1} \wedge u^{\prime \prime}+u_{2} \wedge u^{\prime}, v_{1} \wedge u^{\prime \prime}+v_{2} \wedge u^{\prime}\right\rangle .
$$

In particular, $r=3$.

\section{REFERENCES}

[BF] G. Bagnera and M. de Franchis, Sopra le superficie algebriche che hanno le coordinate del punto generico esprimibili con funzioni meromorfe quadruplemente periodiche di 2 parametri, Rend. Acc. dei Lincei 16, (1907); reprinted in Collected works of Michele de Franchis, Rend. Circ. Mat. Palermo (2) Suppl. No. 27 (1991). MR 93i:01031

[BPV] W. Barth, C. Peters, and A. Van de Ven, Compact complex surfaces, Ergebnisse Math. Grenzgebiete (3) vol. 4, Springer-Verlag, 1984. MR 86c:32026

[Be1] A. Beauville, Surfaces algébriques complexes, Astérisque $n^{\circ} \mathbf{5 4}$ (1978). MR 58:5686

[Be2] A. Beauville, L'application canonique pour les surfaces de type général, Invent. Math. 55 (1979), 121-140. MR 81m:14025

[Br] T. Breuer, Characters and Automorphism Groups of Compact Riemann Surfaces, London Math. Soc. Lecture Note Series 280, Cambridge University Press, 2000.

[Ca] F. Catanese, Fibred Surfaces, varieties isogeneous to a product and related moduli spaces, Amer. Jour. of Math. 122 (2000) 1-44. MR 2002i:14034

[Fu] T. Fujita, On Kaehler fibre spaces over curves, J. Math. Soc. Japan, 30 (1978), 779-794. MR 82h:32024

[K] K. Konno, On the irregularity of special non canonical surfaces, Publ. Res. Inst. Math. Ser., Kyoto Univ. 30 (1994), 671-688. MR 95j:14049

[Pa] R. Pardini, Abelian covers of algebraic varieties, J. reine angew. Math. 417 (1991), 191-213. MR 98g:14012 
[Ser] F. Serrano, Isotrivial Fibred Surfaces, Ann. Mat. Pura Apl. (4), 171, 1996, 63-81. MR 98e: 14036

[To] H. Tokunaga, On dihedral Galois coverings, Canad. Journ. of Math. Vol. 46 (1994), 1299-1317. MR 96d:14015

[X] G. Xiao, Irregularity of surfaces with a linear pencil, Duke Math. J. 55 (1987), 597-602. MR 89c: 14055

[Z] F. Zucconi, Abelian Covers and Isotrivial Canonical Fibrations, Comm. in Alg. 29 (2001), 5641-5671.

Dipartimento di Matematica e Informatica, Università degli studi di Udine, Via delle Scienze, 206, 33100 Udine, Italia

E-mail address: zucconi@dimi.uniud.it 